Efficiency improvement and spectral shift of an organic light-emitting device by attaching a hexagon-based microlens array

This article has been downloaded from IOPscience. Please scroll down to see the full text article.

2008 J. Opt. A: Pure Appl. Opt. 10055302

(http://iopscience.iop.org/1464-4258/10/5/055302)

The Table of Contents and more related content is available

Download details:

IP Address: 140.112.113.225

The article was downloaded on 06/02/2009 at 08:07

Please note that terms and conditions apply. 


\title{
Efficiency improvement and spectral shift of an organic light-emitting device by attaching a hexagon-based microlens array
}

\author{
Mao-Kuo Wei ${ }^{1,4}$, Jiun-Haw Lee ${ }^{2}$, Hoang-Yan Lin ${ }^{2}$, Yu-Hsuan $\mathbf{H o}^{2}$, \\ Kuan-Yu Chen ${ }^{2}$, Ciao-Ci Lin ${ }^{1}$, Chia-Fang Wu' ${ }^{2}$ Hung-Yi Lin ${ }^{3}$, \\ Jen-Hui Tsai ${ }^{3}$ and Tung-Chuan $\mathrm{Wu}^{3}$ \\ ${ }^{1}$ Institute of Opto-Electronic Engineering and Department of Materials Science and \\ Engineering, National Dong Hwa University, Hualien, Taiwan 974, Republic of China \\ ${ }^{2}$ Graduate Institute of Photonics and Optoelectronics and Department of Electrical \\ Engineering, National Taiwan University, Taipei, Taiwan 106, Republic of China \\ ${ }^{3}$ Mechanical and Systems Research Laboratories, Industrial Technology Research Institute, \\ Hsinchu, Taiwan 310, Republic of China \\ E-mail: mkwei@mail.ndhu.edu.tw
}

Received 14 December 2007, accepted for publication 21 February 2008

Published 26 March 2008

Online at stacks.iop.org/JOptA/10/055302

\begin{abstract}
In this paper, we present and analyze the influences of the fill factor and the sag of hexagon-based microlenses on the optical characteristics of an organic light-emitting device (OLED), such as spectral shift, CIE (abbreviation of the French 'Commission internationale de l'éclairage') coordinates, viewing angle dependence, luminous current efficiency and luminous power efficiency. Both the luminous current efficiency and luminous power efficiency of the OLED were found to increase linearly on increasing the fill factor of the microlenses. It is also found that the full width at half maximum (FWHM) of the OLED spectra and CIE coordinates decreased linearly on increasing the fill factor of the microlenses. Besides, the efficiency improvement of the OLED increased with the height ratio of attached microlenses. Compared to the OLED, the luminous current efficiency and luminous power efficiency of the device can be enhanced by $35 \%$ and $40 \%$, respectively, by attaching a microlens array having a fill factor of 0.90 and a height ratio of 0.56 . We also observed blue shifts at different viewing angles when microlens arrays were attached to the OLED, which is evidence that the waveguiding modes are being extracted. In our planar OLED, the peak wavelength blue shifted and the FWHM decreased on increasing the viewing angles, due to the microcavity effect.
\end{abstract}

Keywords: luminous current efficiency, luminous power efficiency, organic light-emitting device, microlens, fill factor, height ratio

(Some figures in this article are in colour only in the electronic version)

\section{Introduction}

Due to their thinness, low power consumption and excellent color performance, organic light-emitting devices (OLEDs)

4 Author to whom any correspondence should be addressed. have been successfully applied to the small- and mediumsized flat panel displays for mobile phones and personal digital assistants (PDAs) in recent years. Therefore, many researchers have put great efforts into further developing and modifying the properties of the relative organic materials, thin film structures, and fabrication techniques for the OLEDs. 


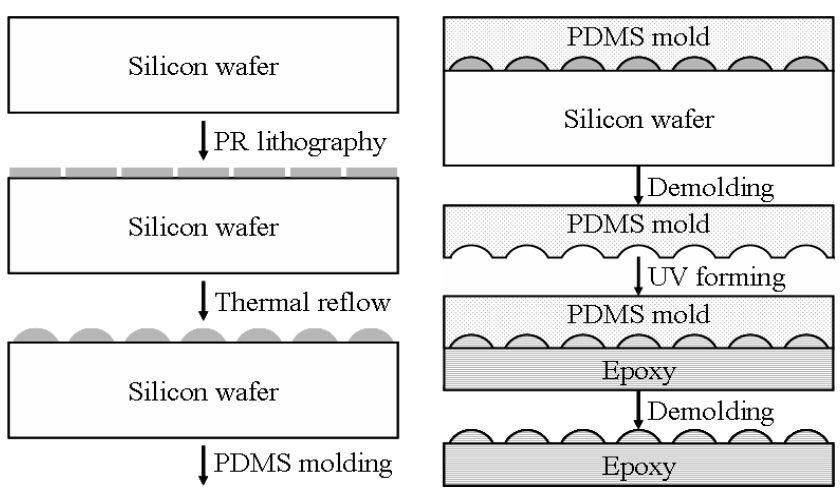

Figure 1. The fabrication process of the microlens array.

Since the refractive indices of the transparent anode and organic thin films are similar and greater than that of the substrate, and the refractive index of the substrate is higher than that of air (1.0), two waveguiding modes, anode/organics and substrate, of light exist in the OLEDs. Thus, only 20$40 \%$ of emitted light of the OLED can propagate into the air. To improve the outcoupling efficiency of the OLEDs, many methods based on eliminating the waveguiding phenomena have been proposed [1-13]. For example, submicron-sized microspheres [1], corrugated and grating-like surfaces [4], photonic crystals [7], micropyramids and V-grooves [3, 13], macrolenses [2], scattering layers [8, 13], low-refractive index airgel films [5], and microlenses [6, 9-12] have been applied to enhance the device efficiency. The macrolenses can strongly enhance the device efficiency, but the devices are too bulky and they violate the development trend of modern electro-optical devices. Microspheres, corrugated surfaces, and photonic crystals will strongly change the optical emitting spectra of the devices at different viewing angles. Besides, the process for airgel films is not compatible with the existing fabrication flow. Of these techniques, the microlens array can improve the moderate efficiency of the OLED, and the fabrication of microlens arrays is simple and reliable in processing [6, 9-12], which is preferable for the industrial mass production.

Until now, both square-based $[6,9,11]$ and roundbased $[10,12]$ microlens arrays have been studied to enhance the outcoupling efficiency of the OLEDs. As illustrated in previous works $[9,11]$, the greater the fill factor of the square-based microlenses, the greater the improvement of the luminous current efficiency of the OLED. Though the squarebased microlens arrays can have higher fill factors than the round-based ones, the round-based microlenses do have more perfectly spherical contours than the square-based ones. To increase the fill factor but not at the cost of the spherical contour too much, a hexagon-based microlens array seems to be a good choice.

In this paper, hexagon-based microlens arrays with different fill factors were used to increase the outcoupling efficiency of the planar OLEDs. According to previous work [11], similar influences of microlens arrays on the efficiency improvement and spectra of OLEDs with different emitting colors were found. Therefore, only a green-color OLED was used. The influence of the fill factor on the

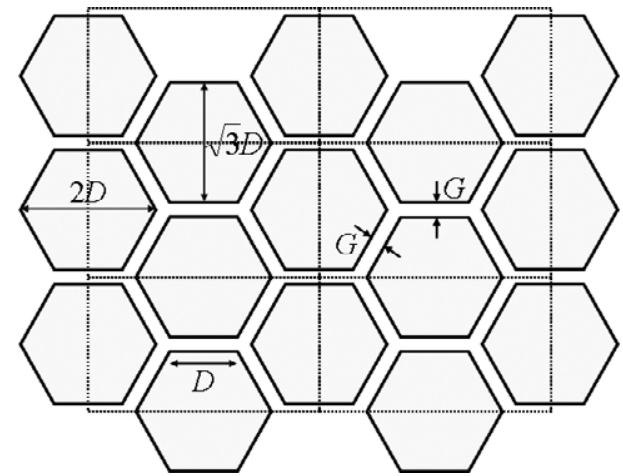

Figure 2. The arrangement of hexagon-based microlenses.

Table 1. The parameters of hexagon-based microlens arrays.

\begin{tabular}{lll}
\hline Set number & HO25 & HI28.9 \\
Shape of base region & Hexagon & Hexagon \\
Edge length of base region, $D(\mu \mathrm{m})$ & 25.0 & 28.9 \\
Thickness of photoresist, $d(\mu \mathrm{m})$ & 8.9 & 8.9 \\
Gap distance, $G(\mu \mathrm{m})$ & $2.3-53.5$ & $2.7-61.7$ \\
Fill factor & $0.200-0.902$ & $0.201-0.900$ \\
Height of microlens, $h(\mu \mathrm{m})$ & $13.5 \pm 0.1$ & $13.5 \pm 0.2$ \\
Radius of curvature of microlens, $r(\mu \mathrm{m})$ & $24.2 \pm 0.2$ & $30.2 \pm 0.1$ \\
Height ratio, $h / r$ & $0.56 \pm 0.01$ & $0.45 \pm 0.01$ \\
\hline
\end{tabular}

optical properties of a green OLED was studied in this paper. In addition, the influences of the height ratio of hexagonbased microlenses on the efficiency improvement and optical properties of the OLEDs were also investigated.

\section{Experimental methods}

Microlens arrays were made by the combination of photolithography, thermal reflow, molding, and UV forming techniques, which is similar but slightly different from the fabrication method given in the previous work [11], in the molding process. The process for the duplication of microlens arrays is depicted in figure 1. First, a 4 inch (100) silicon wafer was used as a substrate. Second, photoresist AZ P4620 (from Clariant K K Technology Production Dept.) was spun on the wafer, and photoresist plates, having hexagonal bases, were made through a photolithography process. The arrangement of these plates is shown in figure 2. The parameters of these plates are listed in table 1 in detail. Third, these plates were put in a furnace to transform them into the shape of spherical caps. The temperature and duration for thermal reflow were set at $230{ }^{\circ} \mathrm{C}$ and $44 \mathrm{~h}$, respectively. Thus, a microlens array template was formed after the thermal reflow process. Fourth, liquid polydimethylsiloxane (PDMS, trade mark Sylgrad 184A) mixed with its hardener (trade mark Sylgrad 184B) was poured onto the wafer and put in an oven to be thermally cured at $65^{\circ} \mathrm{C}$ for $4 \mathrm{~h}$. Fifth, the PDMS mold with a concave microlens array on the mold surface was released by peeling off the wafer and the mold. Compared with the previous electroforming process by using a nickel mold [11], this improved process exhibits the advantages of the re-use of the thermal-reflowed $\mathrm{Si}$ wafer and easy fabrication. Sixth, liquid epoxy OG152 (from Epoxy 
Technology Co.) was coated on the PDMS mold and then exposed to UV radiation at a dose of more than $1 \mathrm{~J} \mathrm{~cm}^{-2}$ to fully harden the epoxy. The hardened epoxy film possesses a refractive index of 1.5303 and is transparent in the visible light range $(>450 \mathrm{~nm})$. Since this refractive index value is close to that of glass, it can effectively eliminate the reflection between the microlens array film and the OLED substrate. Finally, a free-standing epoxy film with the duplicated microlens array on the film surface was formed after separating the film from the mold.

In this study, a green OLED was used as a lighting source; its structure and fabrication process have been described elsewhere $[13,14]$. The thickness of the glass substrate is $0.7 \mathrm{~mm}$ and the active area of the OLED is $10 \times 10 \mathrm{~mm}^{2}$. In our measurement, a source meter (Keithley 2400) was used to supply the driving current for the OLED and to monitor both the current and voltage of the device simultaneously. A spectroradiometer (Minolta CS-1000A) was focused on the active area of the device to measure its luminance, spectrum and CIE coordinates. The resolution of the measured spectra was set at $1 \mathrm{~nm}$. All the instruments were connected to a personal computer and controlled by Labview programs. For the angular-dependent luminance analysis, the device was fixed on a rotational stage, and the luminance was then measured at different viewing angles from $0^{\circ}$ to $85^{\circ}$.

The luminance of the OLED without any microlens arrays was first measured as a reference. Refractive-index-matched oil (Uni-Onward Corp., AK350) with a refractive index of approximately 1.5 was then used to reduce the reflection between the film and the glass substrate of the OLED prior to the optical characterizations. All the microlens arrays were attached in turn to the same OLED device to minimize measuring errors from different OLEDs. To reduce the influence of the ambient light on the CIE coordinates, the luminance of the device without any microlens arrays was set at $\sim 680 \mathrm{~cd} \mathrm{~m}^{-2}$. In this study, all the experiments were executed with the same driving current for the device with and without microlens arrays. Therefore, the driving currents, driving voltages, and active areas of the device were the same in this study. The edge lengths of the base region and the gap distances of the photoresist plates (before thermal reflow) were measured using a surface profiler (Tencor, Alpha-Step 500). The sags of microlenses were measured with a scanning electron microscope (Hitachi S3500).

\section{Results and discussion}

As depicted in figure 2, the fill factor $(F)$ of the hexagon-based photoresist plates (before thermal reflow) can be derived from a simple geometric relationship:

$$
F=\left(\frac{\sqrt{3} D}{\sqrt{3} D+G}\right)^{2}
$$

where $D$ and $G$ represent the edge length of the base region of the microlenses and the gap distance between two adjacent microlenses, respectively. Since there are no obvious changes in the shape and size of the base region of photoresist plates (a)

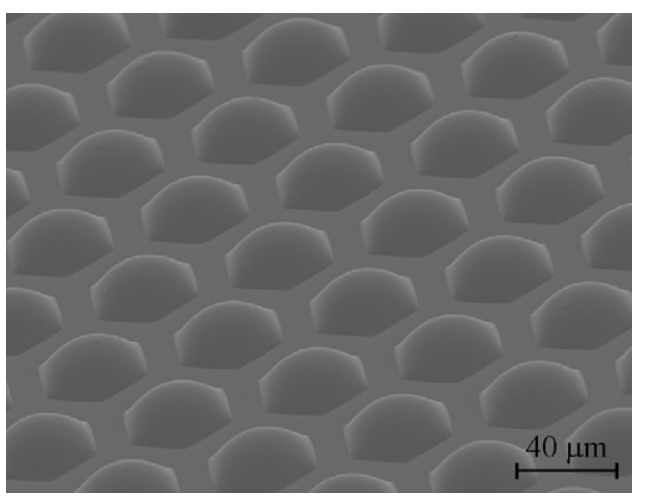

(b)

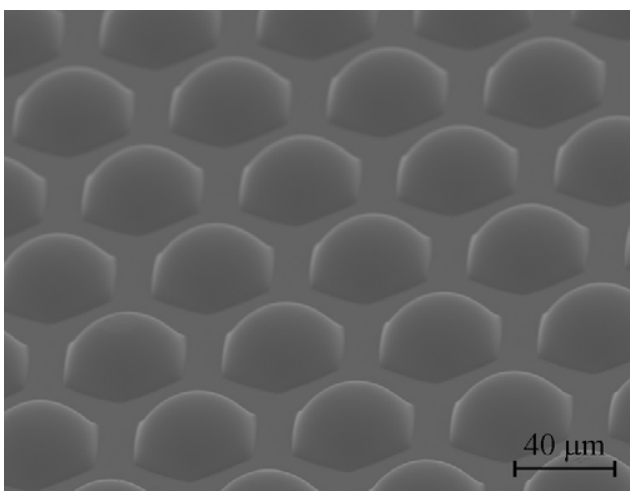

Figure 3. Microphotographs of hexagon-based microlens arrays having edge lengths of (a) 25.0 and (b) $28.9 \mu \mathrm{m}$. (Fill factor: $\sim 0.6$, tilt angle: $\sim 60^{\circ}$.)

before and after thermal reflow process, equation (1) can still be used to calculate the fill factor of microlens arrays. If the gap distance approaches zero, the fill factor of the hexagon-based microlens array can attain nearly $100 \%$. This value is larger than the maximal fill factor of $78.5 \%$ for a regular circular microlens array. Since the extraction efficiency increases with higher fill factor of microlenses, the OLED efficiency may be improved by more than $20 \%$ when attached to a hexagonbased microlens array $(F: 100 \%)$ rather than to a regular circular microlens array $(F: 78.5 \%)$ [9]. Even in the case of a hexagonal array of circular microlenses, the fill factor is enhanced by more than $9 \%$.

Typical duplicated free-standing epoxy films with hexagon-based microlens arrays, having the same fill factor of $\sim 0.60$, on their surfaces are shown in figure 3 . These microlenses revealed nearly perfectly spherical cap contours in shape, regardless of the sags of the microlenses. Since the sags and profiles of microlenses are difficult to be measured with commercial surface profilers, for this study they were measured with a scanning electron microscope (SEM). The radii of the curvature of microlenses were analyzed by curve fitting the microlens contours obtained from the SEM microphotographs. The sags, radii of curvature and height ratios were calculated by averaging the values of more than 10 microlenses, as listed in table 1.

Lin et al [15] have modeled the variations of feature sizes between the photoresist plates (before thermal reflow) and hexagon-based microlens array (after thermal reflow). 
They proposed an approximate solution for this volume transformation based on the volume of a hexagonal photoresist plate being approximately equal to the volume of a spherical cap subtracting cut-off volumes of six corners from the original hexagonal photoresist column. The thickness and the radius of curvature of a hexagonal microlens can be calculated using equations (2) and (3).

$$
\begin{gathered}
t=\frac{2 \sqrt{3} \pi}{27}\left(\frac{h}{D}\right)^{2}(3 r-h)-\left(\frac{16 \sqrt{3}-27}{18}\right)\left(\frac{D^{*}}{D}\right)^{2} h \\
r=\frac{h}{2}+\frac{D^{* 2}}{2 h}
\end{gathered}
$$

where $t$ and $D$ represent the thickness and the edge length of base region of photoresist plates, respectively. $D^{*}, h$ and $r$ are the edge length of the base region, the sag and the radius of curvature of microlenses, respectively. Also, the focal length $(f)$ and numerical aperture (NA) of microlenses can be calculated using equations $(4 a)$ and $(4 b)$ based on optical theory.

$$
\begin{gathered}
\frac{1}{f}=(n-1)\left[\frac{1}{R_{1}}-\frac{1}{R_{2}}+\frac{(n-1) d}{n R_{1} R_{2}}\right] \\
\mathrm{NA}=\frac{R}{2 f}
\end{gathered}
$$

where $n$ and $d$ are the refractive index and thickness of the epoxy film, respectively. $R_{1}$ and $R_{2}$ are the radii of curvature of the two surfaces of the microlenses. $R$ represents the aperture of the microlenses. In this study, the aperture is equal to double the edge length $\left(2 D^{*}\right)$ of microlens base region. If $R_{1}$ is the radius of curvature of the convex surface of the microlenses and equal to $r$, and $R_{2}$ is the radius of curvature of the smooth surface and equal to infinity, equation $(4 a)$ can then be reduced to equation $(4 c)$.

$$
f=\frac{r}{n-1} \text {. }
$$

If the base regions of the photoresist plates do not broaden during the thermal reflow, the edge length of the base region of the photoresist plates will be equal to that of the microlenses. Using the value of $t$ of $8.9 \mu \mathrm{m}$, the values of $h, r, h / r$ and $f$ at various edge lengths of hexagon-based microlenses can be calculated by using equations (2)-(4a), as shown in figure 4 . The sag, radius of curvature and focal length increased, but the height ratio decreased on increasing the edge length of the base region of the microlenses. Compared to the simulated results, the behaviors of our measured sags, radii of curvature, height ratios and focal lengths versus the edge length of the base region of microlenses also had similar trends, as depicted in figure 4 . The numerical apertures of microlenses with edge lengths of 25 and $28.9 \mu \mathrm{m}$ were calculated to be 0.517 and 0.418 , respectively.

When the green OLED without any microlenses was driven at a luminance of $\sim 680 \mathrm{~cd} \mathrm{~m}^{-2}$ normal to the surface, the peak wavelength and the full width at half maximum (FWHM) of the emitting spectrum were 515 and $95.6 \mathrm{~nm}$ respectively. The CIE coordinate of the device was $(0.2742$, 0.5317 ) [13]. The angular-dependent luminance of the OLED (a)

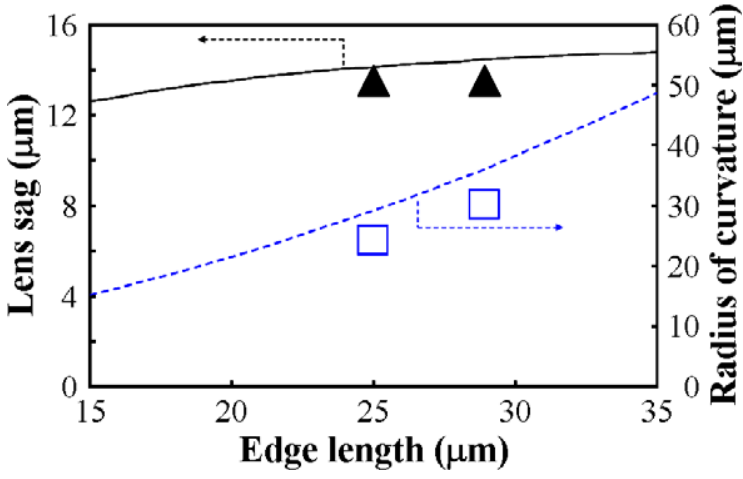

(b)

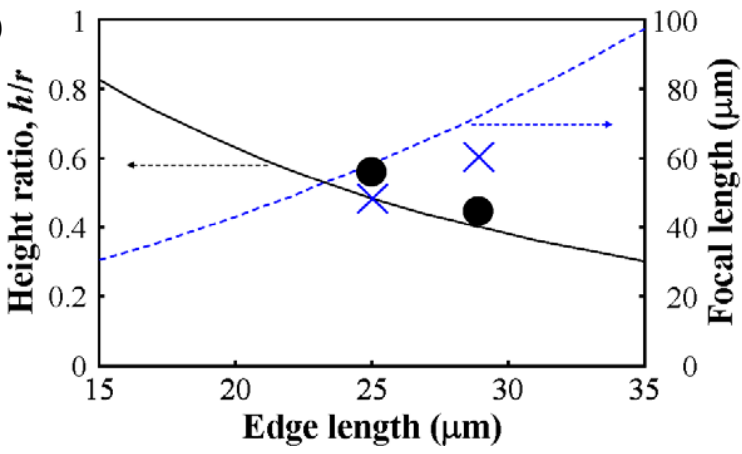

Figure 4. The behaviors of (a) the lens sag and radius of curvature and (b) the height ratio and focal length of microlenses versus the edge length of base region. The solid and dashed lines represent simulated results. The symbols, $\boldsymbol{\Lambda}, \square, \bullet$ and $\times$ represent the measured lens sags, radii of curvature, height ratios and calculated focal lengths of the microlenses.

without microlenses is shown in figure 5. The emission profile of the planar OLED depends on the layer thicknesses of the organic thin films, which can be modeled as a microcavity structure [16-18].

To understand the influences of the fill factor and the height ratio of microlenses on the efficiency enhancement of the OLED, a ray tracing method (LightTools software) was used to simulate the propagation of light rays from a Lambertian OLED device with different microlens parameters. Figure 6(a) shows the cross section of the planar OLED. We can see that the light rays are reflected back at the glass substrate/air interface when the incident angle is larger than the critical angle. On the other hand, in figures 6(b)-(d), the light rays are possibly coupled out even the incident angle is larger than the critical angle. As the fill factor is increased from 0 to 0.2 and then to 0.9 , corresponding to figures 6 (a)(c) respectively, the number of outcoupling light rays increases accordingly. From figures 6(a), (d) and (c), we can find that as the height ratio is increased from 0 to 0.5 and then to 1.0 , the number of outcoupling light rays also increases accordingly.

In this study, microlenses with different fill factors and sags were attached in turn to the same OLED device, and the luminance perpendicular to the device surface was then measured. Since the driving currents, driving voltages, and the active areas of the OLED were kept the same, the luminance ratio of the device with microlenses to that without microlenses should equal the ratio of their luminous current 
(a)

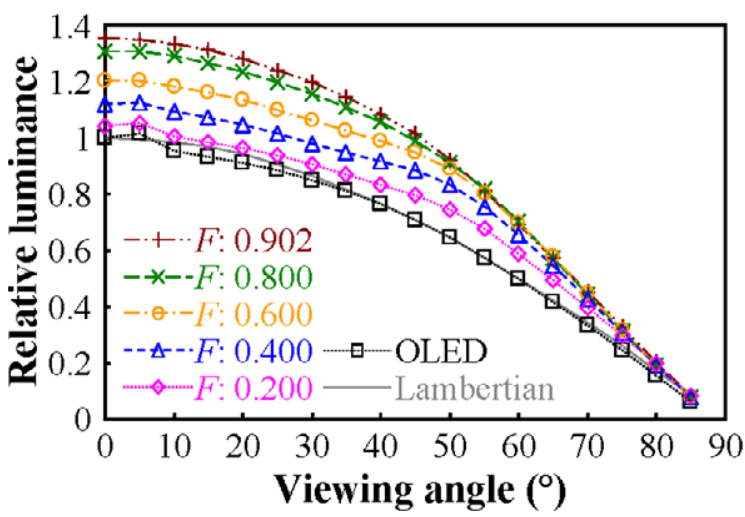

(b)

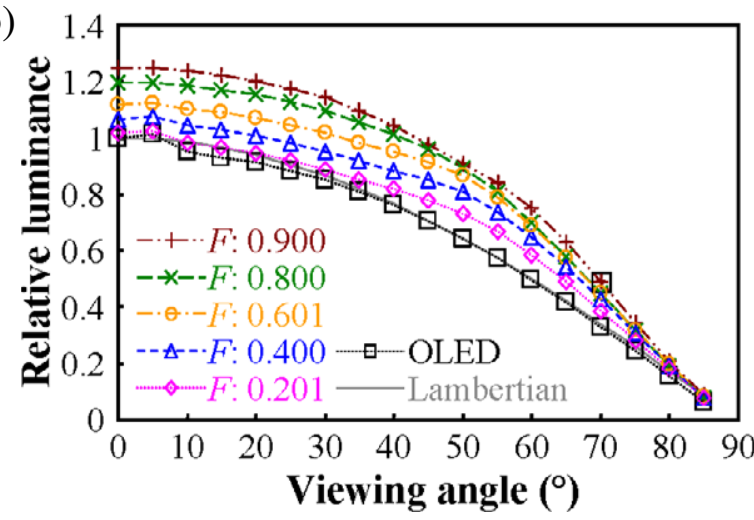

Figure 5. The angular-dependent luminance of the OLED attached to hexagon-based microlens arrays having edge lengths of (a) 25.0 and (b) $28.9 \mu \mathrm{m}$.

efficiencies [9, 13], and the ratio of the luminous power efficiency of the OLED with microlenses to that without microlenses should be equal to the ratio of their luminous existences [13].

The behaviors of the luminance of the OLED with microlenses, having different edge lengths of the base region, versus the viewing angle are described in figure 5. Though the microlenses used had two different sags, the OLED attached to them had similar trends. That is, the luminance of the device decreased with increasing viewing angle, but increased on increasing the fill factor of microlenses. In addition, the attached microlenses with a smaller edge length can enhance more the luminance of the OLED. As illustrated before, microlenses with a larger edge length also possessed a larger sag but a smaller height ratio if the thicknesses of photoresist plates remained the same as before the thermal reflow. In this case, the lens sag alone cannot explain the luminance variation of the OLED since the radius of curvature of the attached microlenses also varies with the edge length. In fact, the height ratio, defined as the ratio of the sag to the radius of curvature, of microlenses is better than both the sag and the edge length for describing the luminance variation of the OLED, since a microlens with a large height ratio also possesses a large curved surface area. Therefore, the luminance of the device increases on increasing the height ratio of the microlenses.

The variation of the luminous current efficiency (normal to the OLED surface) with the fill factor of microlenses is

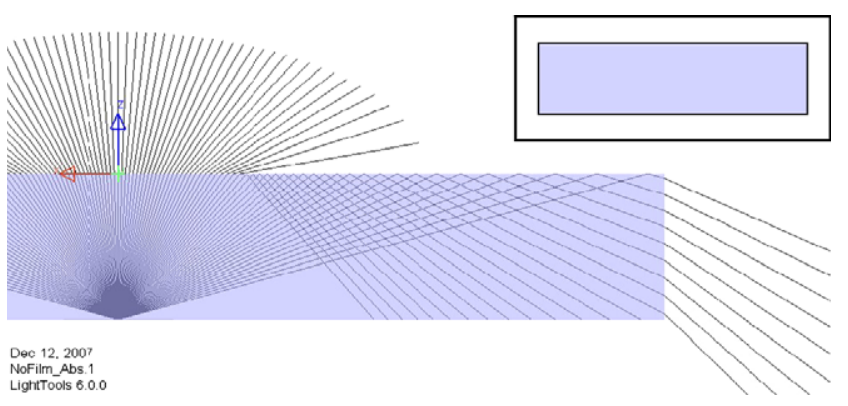

(a)

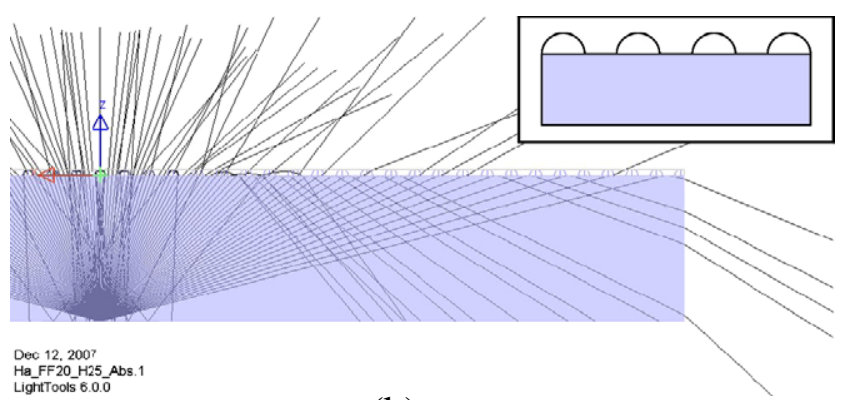

(b)

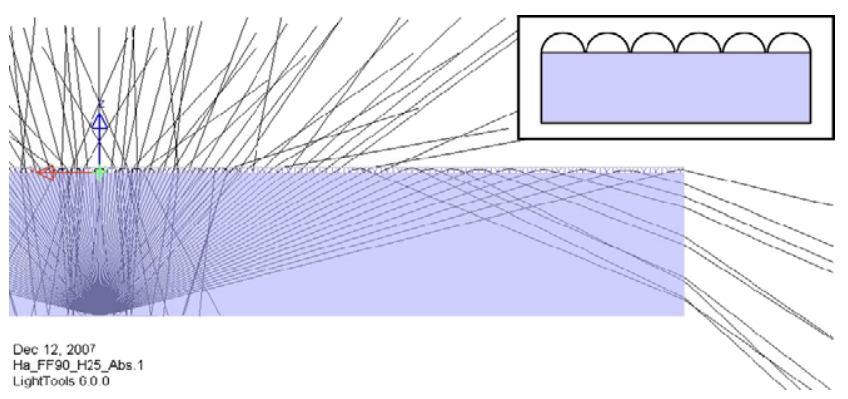

(c)

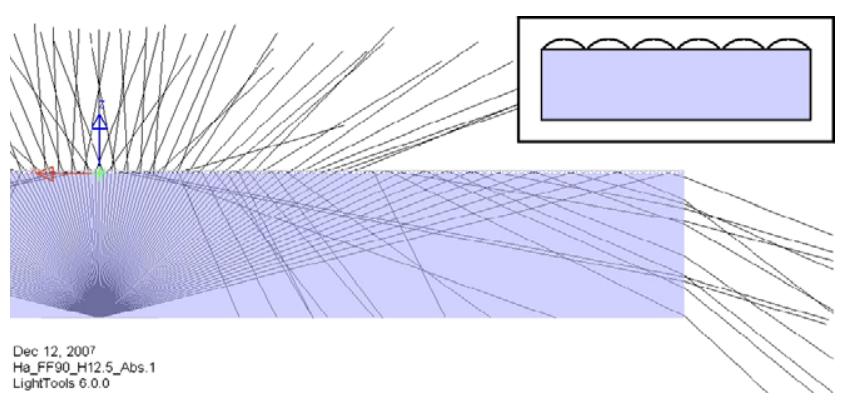

(d)

Figure 6. The ray tracing diagrams of light emitting from the organic layer through the glass substrate: (a) without a microlens array attachment; (b) with a microlens array having a fill factor of 0.2 and a sag of $25 \mu \mathrm{m}$; (c) with a microlens array having a fill factor of 0.9 and a height of $25 \mu \mathrm{m}$; (d) with a microlens array having a fill factor of 0.9 and a height of $12.5 \mu \mathrm{m}$, where the diameter of the microlenses is $50 \mu \mathrm{m}$.

shown in figure 7(a). The luminous current efficiency of the OLED was found to increase linearly with the fill factor of the microlenses. This trend is similar to that in previous works obtained by using square-based microlenses [9, 11]. It also 

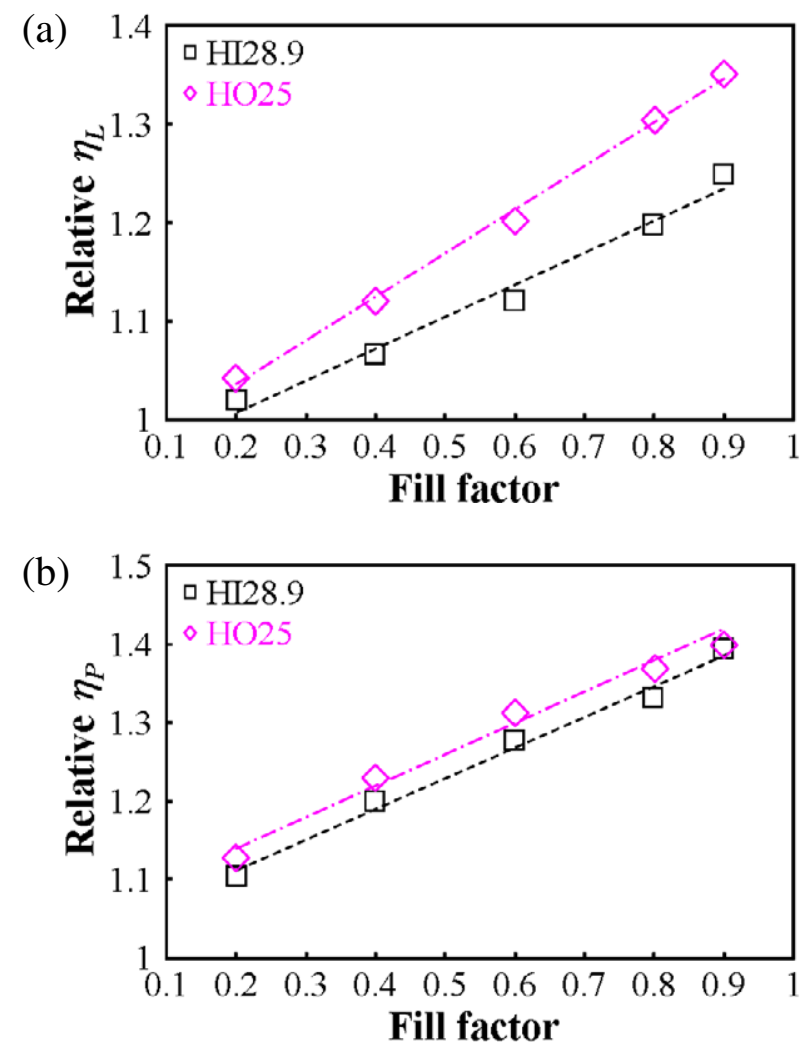

Figure 7. The relationships (a) between the luminous current efficiency and the fill factor and (b) between the power efficiency and the fill factor for hexagon-based microlens arrays with different edge lengths.

showed that the OLED with microlenses having large height ratios had large luminous current efficiencies. Analyzing the data in figure 7(a), proportional relationships between the luminous current efficiencies $\left(\eta_{\mathrm{L}}\right)$ and the fill factors $(F)$ can be formulated:

$$
\frac{\eta_{\mathrm{L}, \text { lens }}}{\eta_{\mathrm{L}, 0}}=\frac{L_{\text {lens }}}{L_{0}}=k_{1} F+k_{2}
$$

where $\eta_{\mathrm{L} \text {, lens }}$ and $\eta_{\mathrm{L}, 0}$ represent the luminous current efficiencies of the OLED with and without microlenses, respectively. $L_{\text {lens }}$ and $L_{0}$ are the luminance of the OLED with and without microlenses, respectively. $k_{1}$ and $k_{2}$ represent the slope and constant in these curves, respectively, as listed in table 2. In fact, $k_{1}$ is the enhancement factor of the luminous current efficiency of the OLED when the substrate surface is fully covered with microlenses [9]. Thus, attached microlenses with a larger height ratio were found to have a larger enhancement factor for the luminous current efficiency of the OLED.

The relationship between the luminous power efficiency of the device with the fill factor of microlens array is depicted in figure 7(b). The luminous power efficiency of the OLED increased as the fill factor and the height ratio of attached microlenses increased. Analyzing the data in figure 7(b), proportional relationships between the luminous
Table 2. The values of $k_{1}, k_{2}, k_{3}$ and $k_{4}$.

\begin{tabular}{lllll}
\hline Set number & $k_{1}$ & $k_{2}$ & $k_{3}$ & $k_{4}$ \\
\hline HO25 & 0.4430 & 0.9467 & 0.4026 & 1.0578 \\
HI28.9 & 0.3241 & 0.9422 & 0.3927 & 1.0329 \\
\hline
\end{tabular}

power efficiencies $\left(\eta_{\mathrm{P}}\right)$ and the fill factors can be found.

$$
\frac{\eta_{\mathrm{P}, \text { lens }}}{\eta_{\mathrm{P}, 0}}=k_{3} F+k_{4}
$$

where $\eta_{\mathrm{P}, \text { lens }}$ and $\eta_{\mathrm{P}, 0}$ represent the luminous power efficiencies of the OLED with and without microlenses, respectively. $k_{3}$ and $k_{4}$ represent the slope and constant in these curves, respectively, as listed in table 2. As the fill factor approaches zero, there are no microlenses on the duplicated epoxy film. Therefore, $k_{4}$ is the enhancement factor of the luminous power efficiency of the OLED when attached to a smooth epoxy film. In fact, the value of $k_{4}$ in our results is close to unity.

If there are no microlenses and duplicated film on the OLED, the value of $k_{4}$ should be unity. If $k_{4}$ equals unity, equation (6) can then be rewritten as

$$
\frac{\eta_{\mathrm{P}, \text { lens }}-\eta_{\mathrm{P}, 0}}{\eta_{\mathrm{P}, 0}}=k_{3} F
$$

Therefore, $k_{3}$ is the enhancement factor of the luminous power efficiency of the OLED when the emitting surface of the device is fully covered with microlenses. The enhancement factor of the luminous power efficiency of the OLED increased as the height ratio of attached microlenses increased.

The spectra of the OLED with the hexagon-based microlens arrays, having the same fill factor of 0.90 and different sags, at different viewing angles are shown in figure 8. A blue shift, compared to the spectrum of the OLED, was observed at the normal direction. The peak wavelength shifted $-3.5 \mathrm{~nm}$ and $-3.0 \mathrm{~nm}$, and the FWHM was narrowed by $-3.7 \mathrm{~nm}$ and $-3.0 \mathrm{~nm}$ when the OLED was attached to microlens arrays having height ratios of 0.56 and 0.45 , respectively. In addition, the spectral shift increased on increasing both the fill factor and the height ratio of the microlenses. Such a spectral shift may be exploited for applications by realizing a device with automatically turning angle or adaptive microlenses (spectral tuning).

The glass substrate in our device acts as a slab waveguide which limits the outcoupling efficiency of the OLED. Typically, light emission from the organic emitter to the external mode can be viewed as a three-step process: first the photons generated in the organic thin films (typically 100 to $200 \mathrm{~nm}$ ) experience an interference effect, then propagate into the thick glass substrate $(0.55$ or $0.7 \mathrm{~mm})$, and finally couple out to the air with a limited extraction efficiency $[19,20]$. Reasons to account for the spectral distributions at different viewing angles of the OLED with and without microlens array attachment may come from: (1) the material dispersion of glass and organic thin films and hence wavelength-dependent angle of total reflection. Since the refractive indices are a decreasing function of the wavelength, the light cone to be extracted from the surface of the substrate will be an increasing function 
(a)

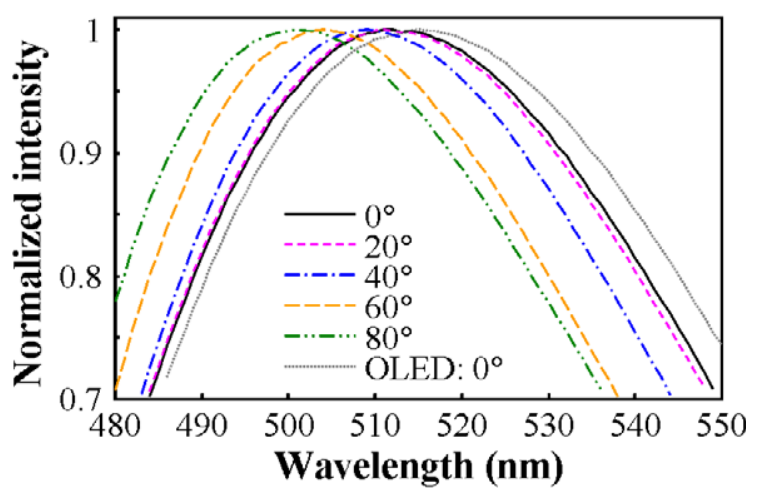

(b)

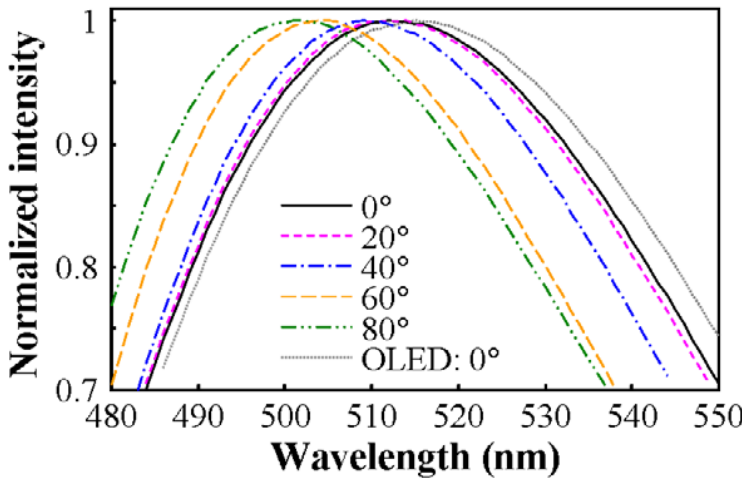

Figure 8. The spectra of the OLED with hexagon-based microlens arrays having edge lengths of (a) 25 and (b) $28.9 \mu \mathrm{m}$ for various viewing angles. The fill factors of both the two microlens arrays are $\sim 0.90$.

of the wavelength. From this viewpoint, there will be red shifts for increasing viewing angles; (2) the intrinsic angular characteristics of the OLED itself. In addition, we observed blue or red shift behaviors when attaching microlens arrays on the OLED depending on their layer structures, i.e., angular characteristics, not shown here. Since the main purpose of this paper is focused on the discussions of optical characteristics of different microlens parameters on the same OLED, this is out of the scope of this paper.

Apart from these spectral shifts, the FWHM decreased linearly on increasing the fill factor of attached microlenses, as illustrated in figure 9. This means that the emitting color of the device gets purer when the OLED is attached to a more densely packed microlens array. The FWHM also decreased on increasing the viewing angle. Analyzing the data in figure 9, proportional relationships between the FWHMs and the fill factors can be formulated:

$$
\mathrm{FWHM}=k_{5} F+k_{6}
$$

where $k_{5}$ and $k_{6}$ represent the slope and constant in these curves, respectively. All the values of $k_{5}, k_{6}$ and the FWHMs of the OLED at different viewing angles are listed in table 3. If the device is attached without any microlenses $(F=0)$, the value of $k_{6}$ should approach the FWHM of the OLED. In our results, the values of $k_{6}$ were close to the FWHM of the OLED. Therefore, $k_{5}$ is the difference of the FWHMs between the OLED without and fully covered with microlenses. As
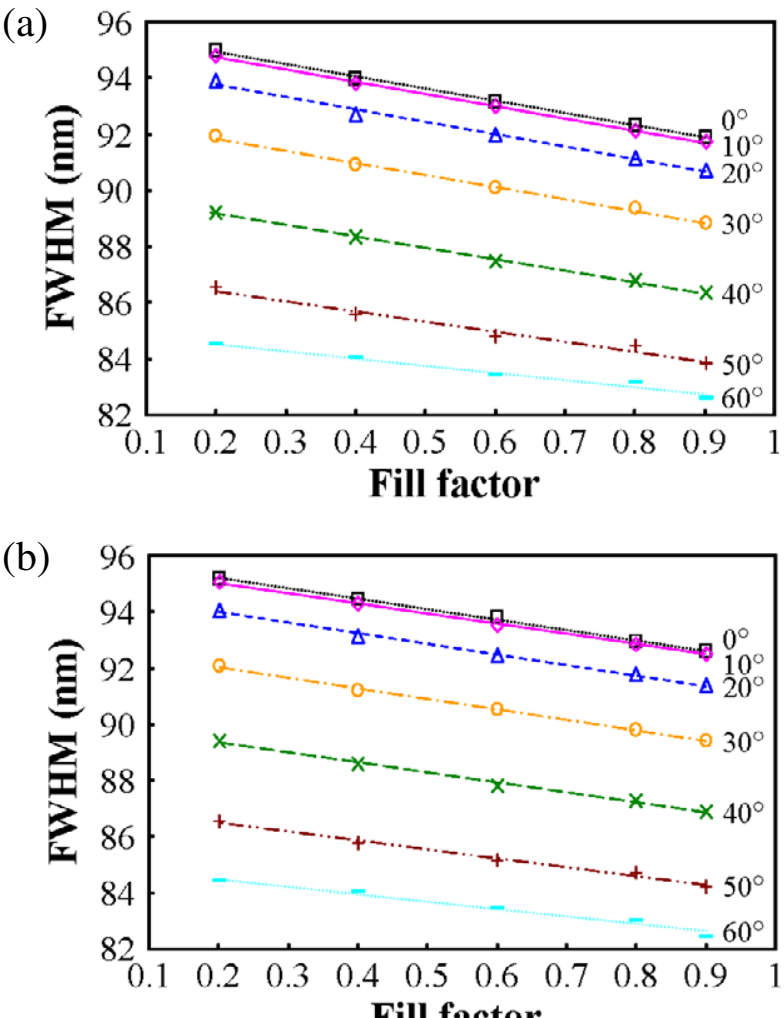

Fill factor

Figure 9. The relationships between the FWHM of the spectra and the fill factor. The edge lengths of hexagon-based microlens arrays are (a) 25 and (b) $28.9 \mu \mathrm{m}$, respectively.

Table 3. The values of $k_{5}, k_{6}$ and the FWHM of the OLED (FWHM $\mathrm{OLED}$ ).

\begin{tabular}{|c|c|c|c|c|c|}
\hline \multirow{2}{*}{$\begin{array}{l}\text { Viewing } \\
\text { angle (deg) }\end{array}$} & \multicolumn{2}{|c|}{$\mathrm{HO} 25$} & \multicolumn{2}{|c|}{ HI28.9 } & \multirow{2}{*}{$\begin{array}{l}\text { FWHM }_{\text {OLED }} \\
(\mathrm{nm})\end{array}$} \\
\hline & $k_{5}$ & $k_{6}$ & $k_{5}$ & $k_{6}$ & \\
\hline 0 & -4.35 & 95.79 & -3.69 & 95.94 & 95.6 \\
\hline 10 & -4.31 & 95.59 & -3.66 & 95.78 & 95.5 \\
\hline 20 & -4.39 & 94.65 & -3.75 & 94.75 & 94.6 \\
\hline 30 & -4.27 & 92.69 & -3.70 & 92.76 & 92.8 \\
\hline 40 & -4.07 & 90.00 & -3.58 & 90.08 & 90.2 \\
\hline 50 & -3.64 & 87.15 & -3.17 & 87.13 & 87.5 \\
\hline 60 & -2.60 & 85.06 & -2.69 & 85.03 & 84.7 \\
\hline
\end{tabular}

observed in table 3 , the larger the height ratio of microlenses, the smaller the value of $k_{5}$. That is, the FWHM decreased on increasing the height ratio of microlenses.

The variations of the CIE coordinates with the fill factor at different viewing angles for the OLED attached with microlenses having height ratio of 0.56 and 0.45 are described in figures 9 and 10, respectively. All the CIE- $x$ and CIE- $y$ indices of the device decreased linearly with increasing the fill factor of microlens arrays. These results also approved the blue shift of the device with microlenses attached. Analyzing the data in figures 10 and 11, proportional relationships between the CIE coordinates and the fill factors can be formulated:

$$
\begin{aligned}
\text { CIE- } x & =k_{7} F+k_{8} \\
\text { CIE- } y & =k_{9} F+k_{10}
\end{aligned}
$$


Table 4. The values of $k_{7}, k_{8}, k_{9}, k_{10}$ and the CIE coordinates of the OLED.

\begin{tabular}{|c|c|c|c|c|c|c|c|c|c|c|}
\hline \multirow{2}{*}{$\begin{array}{l}\text { Viewing } \\
\text { angle (deg) }\end{array}$} & \multicolumn{4}{|c|}{ OLED with microlenses (RO25) } & \multicolumn{4}{|c|}{ OLED with microlenses (RI28.9) } & \multicolumn{2}{|c|}{ OLED } \\
\hline & $k_{7}$ & $k_{8}$ & $k_{9}$ & $k_{10}$ & $k_{7}$ & $k_{8}$ & $k_{9}$ & $k_{10}$ & CIE-X & CIE-y \\
\hline 0 & -0.0114 & 0.2757 & -0.0011 & 0.5329 & -0.0096 & 0.2758 & -0.0008 & 0.5325 & 0.2742 & 0.5317 \\
\hline 10 & -0.0117 & 0.2756 & -0.0013 & 0.5330 & -0.0101 & 0.2758 & -0.0010 & 0.5325 & 0.2743 & 0.5316 \\
\hline 20 & -0.0124 & 0.2739 & -0.0025 & 0.5340 & -0.0110 & 0.2740 & -0.0024 & 0.5338 & 0.2727 & 0.5330 \\
\hline 30 & -0.0131 & 0.2699 & -0.0049 & 0.5357 & -0.0120 & 0.2703 & -0.0049 & 0.5357 & 0.2695 & 0.5348 \\
\hline 40 & -0.0131 & 0.2637 & -0.0085 & 0.5368 & -0.0118 & 0.2639 & -0.0078 & 0.5367 & 0.2643 & 0.5364 \\
\hline 50 & -0.0124 & 0.2561 & -0.0133 & 0.5356 & -0.0114 & 0.2564 & -0.0122 & 0.5359 & 0.2583 & 0.5368 \\
\hline 60 & -0.0115 & 0.2497 & -0.0214 & 0.5326 & -0.0115 & 0.2501 & -0.0202 & 0.5333 & 0.2513 & 0.5357 \\
\hline
\end{tabular}

(a)

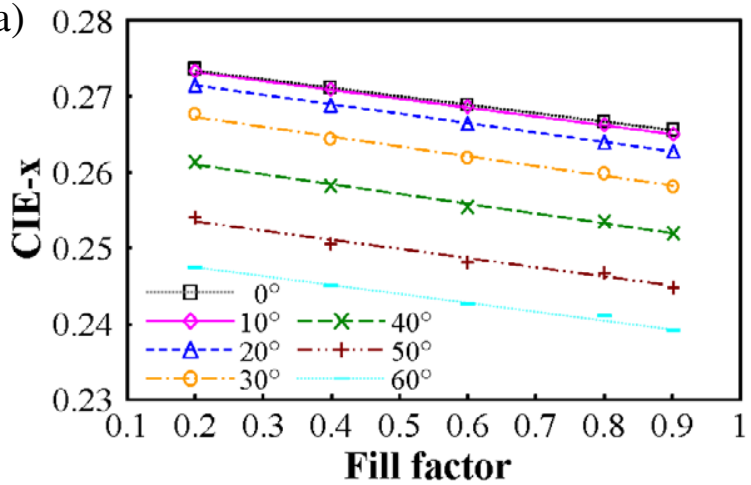

(b)

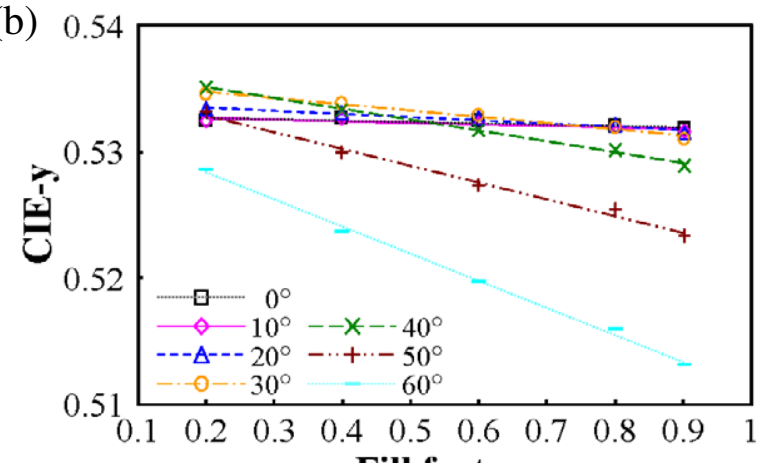

Fill factor

Figure 10. The behaviors of (a) the CIE-x and (b) the CIE-y indices with the fill factor at various viewing angles. The hexagon-based microlens array has an edge length of $25 \mu \mathrm{m}$ and a height ratio of 0.56 .

where $k_{7}$ and $k_{9}$, and $k_{8}$ and $k_{10}$ represent the slopes and constants in the curves of the CIE-x and CIE-y indices versus the fill factor, respectively. All the values of $k_{7}, k_{8}, k_{9}, k_{10}$ and the CIE coordinates of the OLED at different viewing angles are listed in table 4 . As the fill factor approaches zero, $k_{8}$ and $k_{10}$ should approach the CIE-x and CIE-y indices of the OLED without microlenses. This agrees with our results. Thus, $k_{7}$ and $k_{9}$ are the maximal shifts of the CIE-x and CIE-y indices of the OLED when the device is fully covered with microlenses. As shown in table 4 , the values of $k_{7}$ and $k_{9}$ decreased on increasing the height ratio of the microlenses. That is, the greater the height ratio of the microlenses, the more the shift of the CIE coordinates of the OLED.

Since the fill factor is less than unity, the shifts of the CIE- $x$ and CIE-y indices of the OLED should be no more than -0.012 and -0.021 , and -0.011 and -0.020 , for the attached

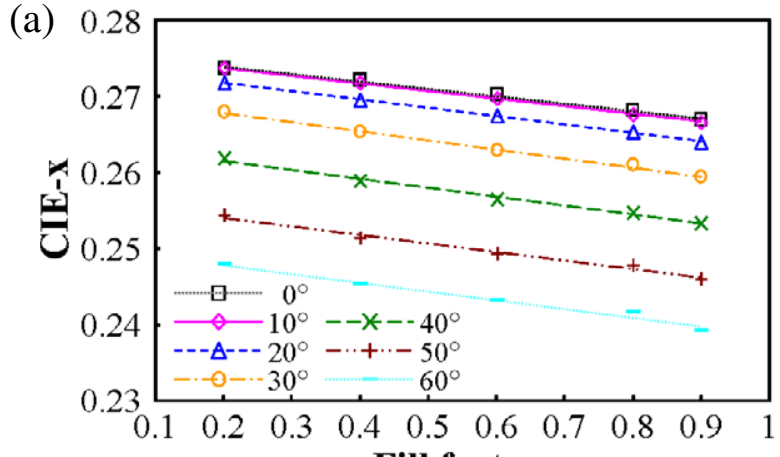

Fill factor

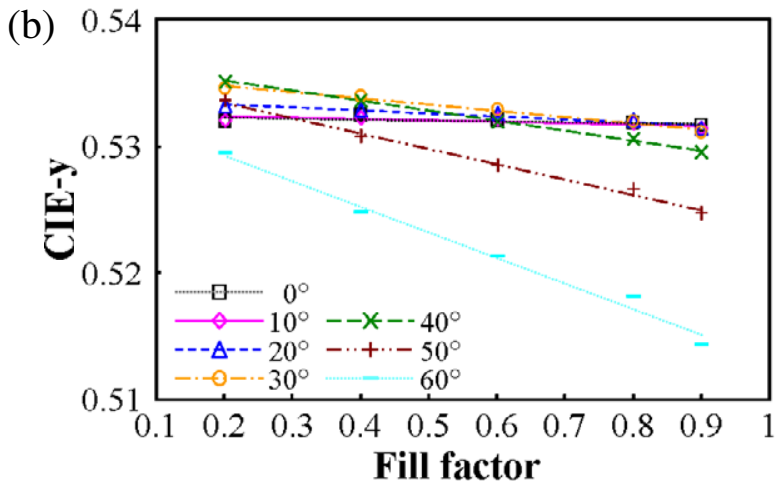

Figure 11. The behaviors of (a) the CIE-x and (b) the CIE-y indices with the fill factor at various viewing angles. The hexagon-based microlens array has an edge length of $28.9 \mu \mathrm{m}$ and a height ratio of 0.45 .

hexagon-based microlenses having height ratios of 0.56 and 0.45 , respectively. These shifts in the CIE coordinates of the OLED are allowable in the industrial specifications for displays and backlights. Therefore, hexagon-based microlens arrays seem to be a good choice for OLED backlight applications.

\section{Conclusions}

Hexagon-based microlens arrays with various fill factors and sags have been produced and studied with the goal of improving the luminous current efficiency and luminous power efficiency of a planar OLED. Results showed that the luminous current efficiency and luminous power efficiency of the OLED have been enhanced by $25 \%$ and $39 \%$, and $35 \%$ and $40 \%$ by attaching hexagon-based microlenses having height ratios 
of 0.45 and 0.56 , respectively. It can be expected to further increase the efficiency of the OLED by increasing the height ratio of the attached microlenses. Results also showed that both the luminous current efficiency and luminous power efficiency of the device increased linearly on increasing the fill factor of the attached microlenses. But all the FWHMs of the spectra and the CIE coordinates of the device decreased linearly on increasing the fill factor of microlenses. In summary, high fill factor and large height ratio of microlens arrays are necessary to increase the efficiency of the OLED. Since the fill factor of hexagon-based microlens arrays can be larger than that of round-based ones, hexagon-based microlenses seem to be better for lighting applications.

\section{Acknowledgments}

The authors gratefully acknowledge the financial support given by the National Science Council of the Republic of China under projects NSC 96-2221-E-002-118, NSC 96-2221-E-002126 and NSC 96-2221-E-259-030. We also acknowledge the financial support given by the Chung-Shan Institute of Science and Technology of the Republic of China.

\section{References}

[1] Yamasaki T, Suminoka K and Tsutsui T 2000 Organic light-emitting devices with an ordered monolayer of silica microspheres as a scattering medium Appl. Phys. Lett. 76 1243-5

[2] Madigan C F, Lu M-H and Sturm j C 2000 Improvement of output coupling efficiency of organic light-emitting diodes with backside substrate modification Appl. Phys. Lett. 76 1650-2

[3] Lin L, Shia T K and Chiu C-J 2000 Silicon-processed plastic micropyramids for brightness enhancement applications J. Micromech. Microeng. 10 395-400

[4] Matterson B J, Lupton J M, Safonov A F, Salt M G, Barnes W L and Samuel I D W 2001 Increased efficiency and controlled light output from a microstructured light-emitting diode Adv. Mater. 13 123-7

[5] Tsutsui T, Yahiro M, Yokogawa H, Kawano K and Yokoyama M 2001 Doubling coupling-out efficiency in organic light-emitting devices using a thin silica aerogel layer Adv. Mater. 13 1149-52

[6] Möller S and Forrest S R 2002 Improved light out-coupling in organic light emitting diodes employing ordered microlens arrays J. Appl. Phys. $913324-7$
[7] Lee Y-J, Kim S-H, Huh J, Kim G-H and Lee Y-H 2003 A high-extraction-efficiency nanopatterned organic light-emitting diode Appl. Phys. Lett. 82 3779-81

[8] Shiang J J, Faircloth T J and Duggal A R 2004 Experimental demonstration of increased organic light emitting device output via volumetric light scattering J. Appl. Phys. 95 2889-95

[9] Wei M-K and Su I-L 2004 Method to evaluate the enhancement of luminance efficiency in planar OLED light emitting devices for microlens array Opt. Express 12 5777-82

[10] Peng H, Ho Y L, Yu X-J, Wong M and Kwok H-S 2005 Coupling efficiency enhancement in organic light-emitting devices using microlens array-theory and experiment J. Display Technol. 1 278-82

[11] Wei M-K, Su I-L, Chen Y-J, Chang M, Lin H-Y and Wu T-C 2006 The influence of a microlens array on planar organic light-emitting devices J. Micromech. Microeng. 16 368-74

[12] Sun Y and Forrest S R 2006 Organic light emitting devices with enhanced outcoupling via microlenses fabricated by imprint lithography J. Appl. Phys. 100073106

[13] Lin H-Y, Lee J-H, Wei M-K, Dai C-L, Wu C-F, Ho Y-H, Lin $\mathrm{H}-\mathrm{Y}$ and $\mathrm{Wu}$ T-C 2007 Improvement of the outcoupling efficiency of an organic light-emitting device by attaching microstructured films Opt. Commun. 275 464-9

[14] Lee J-H, Zhu X, Lin Y-H, Choi W-K, Lin T-C, Hsu S-C, Lin H-Y and Wu S-T 2005 High ambient-contrast-ratio display using tandem reflective liquid crystal display and organic light-emitting device Opt. Express 13 9431-8

[15] Lin C-P, Yang H and Chao C-K 2003 Hexagonal microlens array modeling and fabrication using a thermal reflow process J. Micromech. Microeng. 13 1-7

[16] Lee J-H, Chen K-Y, Hsiao C-C, Chen H-C, Chang C-H, Kiang Y-W and Yang C C 2006 Radiation simulations of top-emitting organic light emitting-devices with two- and three-microcavity structures J. Display Technol. 2 130-7

[17] Chen H-C, Lee J-H, Shiau C-C, Yang C-C and Kiang Y-W 2006 Electromagnetic modeling of organic light-emitting devices J. Lightwave Technol. 24 2450-7

[18] Kim J S, Ho P K H, Greenham N C and Friend R H 2000 Electroluminescence emission pattern of organic light-emitting diodes: implications for device efficiency calculations J. Appl. Phys. 88 1073-81

[19] Krummacher B C, Mathai M K, Choong V, Choulis S A, So F and Winnacker A 2006 General method to evaluate substrate surface modification techniques for light extraction enhancement of organic light emitting diodes J. Appl. Phys. 100054702

[20] Greiner H 2007 Light extraction from organic light emitting diode substrates: simulation and experiment Japan. J. Appl. Phys. 46 4125-37 